\title{
Value of Radiotherapy After Minimally Invasive Surgery in Patients With Stage IA1-IIA1 cervical cancer
}

\section{Wen-qi Liu ( $\square$ liuwenqigx@163.com )}

Department of Radiation Oncology, Second Affiliated Hospital of Guangxi Medical University

\section{Yi-xiu Gan}

Department of Radiation Oncology, Second Affiliated Hospital of Guangxi Medical University

\section{Qing-hua Du}

Department of Radiation Oncology, Second Affiliated Hospital of Guangxi Medical University

\section{Jian Li}

Department of Radiation Oncology, Second Affiliated Hospital of Guangxi Medical University

\section{Ye-ping Wei}

Department of Gynecology, Second Affiliated Hospital of Guangxi Medical University

\section{Xu-wei Jiang}

Department of Radiation Oncology, Second Affiliated Hospital of Guangxi Medical University

\section{Xue-mei Xu}

Department of Radiation Oncology, Second Affiliated Hospital of Guangxi Medical University

\section{Hai-ying Yue}

Department of Radiation Oncology, Second Affiliated Hospital of Guangxi Medical University

\section{Xiang-de Li}

Department of Radiation Oncology, Second Affiliated Hospital of Guangxi Medical University Hui-jun Zhu

Department of Radiation Oncology, Second Affiliated Hospital of Guangxi Medical University

\section{Xue Ou}

Department of Radiation Oncology, Second Affiliated Hospital of Guangxi Medical University

\section{Qiu-lu Zhong}

Department of Radiation Oncology, Second Affiliated Hospital of Guangxi Medical University

\section{Dan-jing Luo}

Department of Radiation Oncology, Second Affiliated Hospital of Guangxi Medical University

\section{Qian-fu Liang}

Department of Radiation Oncology, Second Affiliated Hospital of Guangxi Medical University

\section{Yi-ting Xie}

Department of Radiation Oncology, Second Affiliated Hospital of Guangxi Medical University

\section{Qiang-qiang Zhang}


Department of Radiation Oncology, Second Affiliated Hospital of Guangxi Medical University

\section{Geli Li}

Department of Radiation Oncology, Second Affiliated Hospital of Guangxi Medical University

\section{Yuan-ting Shang}

Department of Radiation Oncology, Second Affiliated Hospital of Guangxi Medical University

\section{Research Article}

Keywords: radiotherapy, patients, LRFS, DMFS

Posted Date: January 8th, 2021

DOI: https://doi.org/10.21203/rs.3.rs-136631/v1

License: (c) (1) This work is licensed under a Creative Commons Attribution 4.0 International License. Read Full License 


\section{Abstract}

To estimate whether adjuvant radiotherapy is necessary for patients with stage IA1-IIA1 cervical cancer after laparoscopic hysterectomy, 221 patients were retrospectively analyzed. Sixty-two of them were treated with laparoscopic hysterectomy and adjuvant radiotherapy (group A), 115 underwent open surgery (group B) and 44 received laparoscopic hysterectomy alone (group C). Results showed that the 3year local recurrence-free survival (LRFS) rates of group A, B and $C$ were $98.4 \%, 97.4 \%$ and $86.4 \%$, respectively. The LRFS rates of group $A$ and $B$ surpassed $C$ ( $A$ vs. $B, p=0.634 ; A$ vs. $C, p=0.011 ; B$ vs. $C, p$ $=0.006)$. The inter-group differences of 3-year overall survival (OS) and distant metastasis free survival (DMFS) were not statistically significant. In subgroup analysis of stage IB disease, the 3-year LRFS rates of group A, B and C were $100 \%, 98.8 \%$ and $83.1 \%$, the 3 -year OS rates of group A, B and C were $100 \%$, $98.9 \%$ and $91.5 \%$, respectively. The 3-year LRFS and OS rates of group A and B were significantly superior to group $C(p<0.05)$. Our findings suggest that adjuvant radiotherapy can reduce the risk of recurrence for women with early-stage cervical cancer after laparoscopic hysterectomy and bring survival benefits for patients with stage IB disease.

\section{Introduction}

According to National Comprehensive Cancer Network (NCCN) clinical guidelines, postoperative adjuvant radiotherapy is generally not required for stage IA1 IB cervical cancer patients if there are no high-risk factors (such as lymph-node involvement, nerve invasion, and large tumor) or the intermediate risk factors do not meet the Sedlis criteria. Recently, A recent study by Ramirez, a highly noteworthy phase III study, was published in the October 2018 New England journal of medicine, which found that the 4.5-year disease-free survival and 3-year tumor-free survival in the minimally invasive surgery group were significantly lower than those in the open surgery group, and the risk of death or recurrence in the minimally invasive group was 3.74 times higher than that in the open surgery group ${ }^{1}$. A retrospective study by Melame et al. had similar results. It can be speculated that minimally invasive surgery may bring the risk of local recurrence ${ }^{2}$. Therefore, we speculate that minimally invasive hysterectomy for early cervical cancer carries a risk of local failure, it is worth studying whether additional postoperative radiotherapy is needed for these patients with minimally invasive surgery.

\section{Methods}

\section{Ethics approval and consent to participate}

Our protocol were approved by the Ethical Review Committee of the Second Affiliated Hospital of Guangxi Medical University, and all methods were carried out in accordance with relevant guidelines and regulations. Due to the retrospective nature of the study, a waiver of informed consent was approved by the Ethical Review Committee of the Second Affiliated Hospital of Guangxi Medical University. 
From January 2013 to December 2016, a total of 221 patients with early-stage squamous-cell carcinoma, adenocarcinoma, or adenosquamous carcinoma s carcinoma of cervical cancer admitted to our institution. All the women had "low-risk"early-stage cervical cancer which was defined as: patients have one or more intermediate-risk factors after surgery, but the combination of intermediate-risk factors did not meet the Sedlis criteria. The clinical data of eligible patients with stage IA1 (lymph-vascular invasion)IIA1 cervical cancer were retrospectively analyzed. All patients had an Eastern Cooperative Oncology Group (ECOG) performance-status score of 0 or 1 . Exclusion criteria included a history or contraindication to radiotherapy; the advanced stage cervical cancer; absence of severe mental disorders or severe diseases of heart, liver, lung, kidney; the existence of high-risk factors (lymph-node involvement, parauterine invasion, and positive vaginal resection margin). Patients were also excluded if the postoperative pathologic risk factors meet the Sedlis Criteria of the latest version of NCCN Guidelines (Version 1.2020). According to different treatment approaches, patients were assigned to different groups. The first group underwent laparoscopic hysterectomy combined with postoperative radiotherapy (group $A, n=62$ ), the second group only received open surgery (group $B, n=115$ ), and the third group received laparoscopic hysterectomy alone (group $\mathrm{C}, \mathrm{n}=44$ ). The median age was 47 years (24-69 years). Patients were restaged based upon International Federation of Gynecology and Obstetrics (2018 FIGO) Surgical Staging of Cancer of the Cervix Uteri (2018). Enrolled patients underwent open surgery or laparoscopic hysterectomy.

The patients of group A received post-operative radiotherapy carried out by intensity modulated radiotherapy (IMRT). According to the consensus guidelines of the Radiotherapy Oncology Group (RTOG) 0418 and its atlas, the clinical target volume (CTV) consisted of central vaginal CTV and a regional lymph node CTV, includes proximal vaginal, paraginal tissues, the internal and external iliac, and anterior sacral lymph nodes. The planned target volume (PTV) is generated by homogeneous expansion of CTV at $7 \mathrm{~mm}$. Plan design based on anatomical boundaries. These boundaries are: Superior-L5 / S1; Lowerbottom of the obturator foramen; the lateral edge of the pelvis was $2 \mathrm{~cm}$, which was adjusted according to the vascular contour. The first $5 \mathrm{~mm}$ of the symphysis pubis should be adjusted according to the vascular contour; and the back-S2 / S3. The organs at risk (OAR) profile include bladder, bowel cavity, rectum, femur head, and other normal tissues. The prescription dose of PTV is 45-50 Gy, 1.8-2.0 Gy per fraction 5 times a week carried out in 5 weeks. The goal of plan for IMRT is to obtain $95 \%$ of the prescribed dose to cover $100 \%$ of PTV, with the maximum dose not exceeding $110 \%$. The maximum dose of OAR limiting dose defined by $2 \%$ (D2 \%) of the maximum tissue dose was 50 Gy. Supplementary restriction V50 Gy (volume exposed to radiation of $50 \mathrm{~Gy}$ ): rectum < 40\%, bladder $<50 \%$, femoral head < $5 \%$, V20Gy $<30 \%$ and V30Gy $<20 \%$ for intestine. None of the patients received concurrent chemotherapy.

SPSS 24.0 statistical software was used for data analysis. The primary outcome was 3-year LRFS rates. The secondary endpoints were 3-year OS and 3-year DMFS rates. Survival rates were used to calculate by Kaplan-Meier method. Cox regression analysis was used to estimate prognostic factors. And the statistically significant $P$ value was a two-tailed $P$ value less than 0.05 . 


\section{Results}

\section{Characteristics of patients}

The clinical baseline characteristics of the three groups including age,tumor size[histologic subtypes, stage of disease and ECOG performance-status score were not statistically different (Table 1). The median age was 47 years (range, 24-69 years). All patients were restaged according to FIGO 2018. The percentage of lymph-vascular invasion was $6.5 \%(n=4)$ in group $A, 1.7 \%(n=2)$ in group $B$ and $2.3 \%(n=$ $1)$ in group $C$. There was no significant difference in the rate of lymph-vascular invasion between-groups. 
Table 1

baseline characteristics of patients

No.of patients (\%)

P-value

Group $A(n=62) \quad$ Group $B(n=115) \quad$ Group $C(n=44)$

Age (years)

Median (range)

$50(27-64)$

$47(24-64)$

$49(24-69)$

0.277

$\leq 47$

$30(48.4)$

63(54.8)

18(40.9)

$>47$

32(51.6)

$52(45.2)$

26(59.1)

Tumor size $(\mathrm{cm})$

$\leq 2$

16(25.8)

28(24.3)

$9(20.5)$

$>2$

46(74.2)

87(75.7)

35(79.5)

Stage of disease

IA1 (lymphovascular invasion) 4(6.5)

$8(7.0)$

2(4.5)

IA2

2(3.2)

$8(7.0)$

$3(6.8)$

IB1

34(54.8)

79(68.7)

35(79.5)

IB2

14(22.6)

10(8.7)

2(4.5)

IB3

2(3.2)

$5(4.3)$

1(2.3)

IIA1

6(9.7)

$5(4.3)$

1(2.3)

Histologic subtype - no. (\%)

0.150

Squamous-cell carcinoma

Adenocarcinoma

$50(80.6)$

90(78.3)

34(77.3)

Adenosquamous carcinoma

10(16.1)

25(21.7)

$8(18.2)$

ECOG performance-status score

2(3.2)

$0(0)$

2(4.5)

0

0

1

\section{Primary Outcome}

With a median follow-up time of 58.33 months (range, 56.90 to 59.76 months), the 3-year LRFS, DMFS and OS rates for all patients were $95.5 \%, 96.4 \%$ and $96.4 \%$, respectively. A total of 10 relapses occurred in 3 years for all patients. There was one patients encountered local failure and the 3-year LRFS rates were 
$98.4 \%$ in group A. Three patients encountered pelvic or vaginal recurrences and the 3-year LRFS rates were $97.4 \%$ in group $B$. There were 5 patients suffered from the local recurrences and the 3-year LRFS rate was $86.4 \%$ in group $C$, which was significantly lower than that in group $A$ and $B$ ( $A$ vs. $B, p=0.634$, A vs. C, $p=0.011, B$ vs. $C, p=0.006)$ ( Table 2 and Fig. 1 ).

Table 2

Incidence and pattern of cervical cancer failures in the three groups

\begin{tabular}{|llll|}
\hline \multicolumn{4}{|c|}{ No. of patients (\%) } \\
\hline Pattern of failures & Group A $(\mathrm{n}=62)$ & Group B $(\mathrm{n}=115)$ & Group C $(\mathrm{n}=44)$ \\
\hline Total failures & $4(6.5)$ & $5(4.3)$ & $6(13.6)$ \\
\hline Pelvis recurrence & 0 & $2(1.7)$ & $2(4.5)$ \\
\hline Vaginal vault & 0 & $1(0.8)$ & $3(6.8)$ \\
\hline Distant metastasis & $3(4.8)$ & $2(1.7)$ & \\
\hline Pelvic recurrence + di & $1(1.6)$ & & $1(2.3)$ \\
stant metastasis & & & \\
\hline
\end{tabular}

\section{Secondary Outcomes}

A total of 7 patients developed lung or bone metastases during 3 years (Table 2). Four of them were in group A, 2 were in group B, 1 was in group C. The 3 -year DMFS rates were $93.5 \%$ in group $A, 98.3 \%$ in group $B, 97.7 \%$ in group $C$, respectively. There was no statistically significant between-group difference (A vs. $B, p=0.123$, A vs. $C, p=0.381$, B vs. $C, p=0.810$ ) (Fig. 2).

Of the 221 patients, 8 patients died within 3 years, all of whom were tumor-related deaths. The 3-year OS rates were $96.8 \%$ in group $A, 97.4 \%$ in group $B$, and $93.2 \%$ in group $C$, respectively. The inter-group differences were not statistically significant ( $A$ vs. $B, p=0.872$, A vs. $C, p=0.341$, $B$ vs. $C, p=0.206$ ) (Fig. 3).

\section{Subgroups Analysis}

In exploratory subgroup analysis of the different stages, we compared the LRFS, DMFS and OS rates across the subgroup of stage IA disease, the subgroup of stage IB1-IB3 disease and the subgroup of stage IIA1 disease, respectively.

In subgroup of stage IA disease, there was one patients (6.2\%) encountered recurrence and death from cervical cancer in group B, the 3-year LRFS and OS rates were both $93.8 \%$. There were not any recurrences and death occurred in group $A$ and group $C$. There was no statistically significant between-group 
difference on 3-year LRFS and OS (group A vs. $B, p=0.540$, group B vs. $C, p=0.576$ ). No patients with stage IA disease had distant metastasis.

In subgroup of stage IB1-IB3 disease, no recurrences and death occurred in group A. Recurrence occurred in one patients in group B and six in group C. The 3-year LRFS was $98.8 \%$ in group B and $83.1 \%$ in group $C$, respectively. The rates of LRFS significantly differed between the three groups (group $A$ vs. $B, p=0.446$; group A vs. C, $p=0.003$; group B vs. $C, p=0.000$ ) (Fig. 4). The 3-year DMFS rates were $96.0 \%$ in group $A$, $98.9 \%$ in group $B, 97.0 \%$ in group $C$. The inter-group differences of DMFS were not different from each other (group $A$ vs. $B, p=0.276$, group $A$ vs. $C, p=0.829$, group $B$ vs. $C, p=0.483$ ). The 3 -year $O S$ rates were $100 \%$ in group $A, 98.9 \%$ in group $B, 91.5 \%$ in group $C$. The significant differences existed between groups (group A vs. $B, p=0.448$, group A vs. $C, p=0.037$, group $B$ vs. $C, p=0.037$ ) (Fig. 5). The LRFS and OS benefits were observed in patients with cervical cancer stage IB1-IB3.

In subgroup of stage IIA1 disease, two patients relapsed, one in group $A(1 / 6)$, another in group $B(1 / 5)$. Two women had distant metastasis, one of them in group $A(1 / 6)$ and another in group $B(1 / 5)$. In terms of OS, a total of three patients died for the disease, two patients in group A, and one in group C. However, the only one patients with stage IIA1 cervical cancer in group C did not had distant metastasis or recurrence. The 3-year LRFS, DMFS and OS rates did not differ significantly between the three approaches $(p>0.05)$.

\section{Prognostic Factors}

Univariate analysis suggested that patients underwent laparoscopic hysterectomy alone had a higher rate of local recurrence than patients received laparoscopic hysterectomy combined with adjuvant radiotherapy or open surgery (hazard ratio for local recurrence, $11.39 ; 95 \% \mathrm{Cl}, 1.36$ to 95.50 ), Multivariate analysis found that a difference remained after the adjustment for, ECOG performance-status score, stage of disease, age, and lymph-vascular invasion (LVSI) (hazard ratio for disease local recurrence from cervical cancer, $12.27 ; 95 \% \mathrm{Cl}, 1.34$ to 112.58). Univariate analysis suggested that deep stromal invasion (DSI) was an independent risk factor for local recurrence (hazard ratio, $3.48 ; 95 \% \mathrm{Cl}, 1.17$ to 10.37 ), multivariate analysis found that a difference remained with the adjustment for ECOG performance-status score, stage of disease, age, and different approaches (hazard ratio for local recurrence, $4.00 ; 95 \% \mathrm{Cl}$, 1.07 to 14.99$)$.

\section{Toxicities Of Postoperative Radiotherapy}

No grade 3 or 4 acute adverse reactions occurred in patients received postoperative radiotherapy. The incidence of grade 1 and 2 adverse reactions of bladder were $12.9 \%$ and $4.8 \%$, respectively. The rates of grade 1 and 2 acute gastrointestinal adverse reactions were $27.4 \%$ and $9.7 \%$, respectively. The incidence of grade 1 and grade 2 hematologic toxicities were $33.9 \%$ and $8.1 \%$, respectively. No acute adverse reactions were observed in the femoral head (Table 3). 
Table 3

The incidences of acute adverse reactions of group A

\begin{tabular}{|lll|}
\hline Acute adverse reactions & Grade I $\mathbf{n}(\%)$ & Grade II n(\%) \\
\hline Intestinal reaction & $17(27.4)$ & $6(9.7)$ \\
\hline dermatitis & $5(8.1)$ & 0 \\
\hline Bladder reaction & $8(12.9)$ & $3(4.8)$ \\
\hline Hematologic toxicity & $21(33.9)$ & $5(8.1)$ \\
\hline
\end{tabular}

\section{Discussion}

In this retrospective analysis, women undergoing laparoscopic hysterectomy combined with adjuvant radiotherapy or open surgery for early-stage cervical cancer had higher 3-year LRFS rates than patients who received laparoscopic hysterectomy alone. For patients with early-stage cervical cancer without any high risk factors after surgery, a prospective study conducted by DELGADO et al. ${ }^{3}$ found that lymphvascular space invasion (LVSI), deep stromal invasion (DSI), and tumor size $\geq 2 \mathrm{~cm}(T S \geq 2 \mathrm{~cm}$ ) were the risk factors for recurrence. And then they put forward the classic standard of adjuvant treatment for early stage cervical cancer: there are at least two intermediate risk factors ( $\operatorname{LVI}(+), D S I>1 / 3, T S \geq 2 \mathrm{~cm}$ ). However, this trial were carried out from March 1981 to February 1984,all patients undergo radical hysterectomy while laparoscopic hysterectomy had not been used. The evaluation of postoperative risk factors was limited in patients with squamous cell carcinoma. SEDLIS then combined intermediate risk factors to form the Sedlis standard ${ }^{4}$. However, according to the study by RYU et al. ${ }^{5}$, the sensitivity of the Sedlis criteria was only $50 \%$, and its scoring method was too complicated and inconvenient for clinical application. In short, there is still controversy regarding the indications for postoperative adjuvant treatment in early stage cervical cancer with intermediate risk factors. Although the NCCN guidelines ${ }^{6}$ recommend the use of Sedlis criteria, different countries and regions still use different standards.

In 1989, Reich H. et al. ${ }^{7}$ reported the world's first laparoscopic hysterectomy. With the continuous improvement of surgical methods, the clinical benefits of laparoscopic hysterectomy for cervical cancer have been affirmed by many studies. Study by Nam JH et al. ${ }^{8,9}$ compared the survival of patients with early stage cervical cancer between laparoscopic radical hysterectomy group and open radical hysterectomy group. They found that compared with open radical hysterectomy $(n=263)$, laparoscopic radical hysterectomy $(n=263)$ did not have higher risks of recurrence [hazard ratio $(H R)=1.28 ; 95 \%$ confidence interval $(\mathrm{Cl}) 0.62-2.64]$ or death $(\mathrm{HR}=1.46 ; 95 \% \mathrm{Cl} 0.62-3.43)$. Even in patients with tumors > $2 \mathrm{~cm}$ in diameter, the risks of recurrence $(\mathrm{HR}=0.82 ; 95 \% \mathrm{Cl} 0.31-2.16)$ or death $(\mathrm{HR}=1.01 ; 95 \% \mathrm{Cl} 0.35-$ 2.95) were not higher for laparoscopic radical hysterectomy than for open radical hysterectomy. The laparoscopic radical hysterectomy and open radical hysterectomy group had 5-year recurrence-free survival rates of $92.8 \%$ and $94.4 \%$, respectively $(P=0.499)$. In the meta-analysis of Yan-zhou Wang et al. 10 , patients who underwent laparoscopic hysterectomy had a lower incidence of postoperative adverse 
reactions. And the 5-year overall survival (HR 0.91, $95 \% \mathrm{Cl} 0.48-1.71 ; \mathrm{p}=0.76)$ and 5-year disease-free survival (hazard ratio $[\mathrm{HR}] 0.97,95 \% \mathrm{Cl} 0.56-1.68 ; \mathrm{p}=0.91$ ) rates of the two groups were not significant different. However, these studies were all retrospective studies and the simple size of the studies included in the meta-analysis were too small. Another meta-analysis regarding laparoscopic hysterectomy and open surgery in 2015 by Cao T et al. ${ }^{11}$ reached the same conclusion. However, there was only one randomized controlled trial with 30 patients included. The other 21 studies were all retrospective or prospective studies with a small simple size and a short follow-up time. In general, the above researches showed that compared with open surgery, laparoscopic hysterectomy did not reduce the overall survival and disease-free survival ${ }^{12,13}$, but also had the advantages of a decrease in operative blood loss, a shorter hospital stay, and a lower rate of postoperative complications than open radical hysterectomy ${ }^{11}$, 14,15 . However, these retrospective studies may have a bias of case mismatch. Because in clinical practice, surgeons will choose cases with earlier stage or smaller lesions to perform laparoscopic hysterectomy. For the other more difficult cases, open surgery was their preferred choice. In November 2018, the New England Journal of Medicine published two results on the comparison of open surgery and minimally invasive surgery. One was a multi-center, prospective, randomized controlled clinical trial (LACC) ${ }^{1}$, and the other was a retrospective epidemiological study ${ }^{2}$. These two studies compared the recurrence and survival results of patients with early cervical cancer undergoing open surgery and minimally invasive surgery, and found that the survival of patients in the open surgery group was significantly better than that of the minimally invasive surgery group, which completely contrary to previous results ${ }^{8-15}$. The same results were obtained in subsequent studies by other institutions ${ }^{16,17}$. It caused great shock and controversy in the international community. Therefore, it can be speculated that the risk factors for early cervical cancer may have changed under the new surgical method of minimally invasive surgery. Although the meta-analysis by Cao T et al. ${ }^{11}$ has balanced various risk factors, the results of this analysis did not reflect the adverse effects of laparoscopic hysterectomy caused on survival of cervical cancer. Based on previous researches' conclusions, the risk factors for early stage cervical cancer after minimally invasive surgery have not been fully explored in the past thirty years. Whether the Sedlis criteria is still suitable for early stage cervical cancer is worthy of further thinking and exploration.

In our study, patients who underwent laparoscopic hysterectomy of cervical cancer had a lower 3-year LRFS than the other two groups. The result is the same as the Laparoscopic Approach to Cervical Cancer (LACC) Trial ${ }^{1}$. The 62 patients in group B did not fully comply with the Sedlis criteria. As long as there were single or two intermediate risk factors, they all received the treatment of laparoscopy + postoperative radiotherapy, and obtained the same survival as open surgery group, indicating that for patients with early cervical cancer, it is not enough to use Sedlis criteria to assess risk factors and guide postoperative adjuvant treatment. Adjuvant treatment is necessary for early cervical cancer after laparoscopic hysterectomy for patients with early stage cervical cancer. At the same time, our result also confirms from the side that the conclusion of LACC Trial that minimally invasive radical hysterectomy was associated with lower rates of disease-free survival and overall survival than open abdominal radical hysterectomy may be still applicable to "low-risk" patients with early stage cervical cancer. The indications for 
postoperative adjuvant treatment are still being explored, but a complete consensus has not yet been formed. Different regions still use different standards. JSGO ${ }^{18}$ recommends that patients with one or more intermediate risk factors can be treated with radiotherapy. However, the establishment of JSCO standards did not recognize the negative impact of laparoscopy on survival, and its use has the risk of overtreatment. The European Society of Medical Oncology (ESMO) ${ }^{19}$ recommends that patients with intermediate risk factors for early stage cervical cancer do not need any adjuvant treatment after surgery (evidence level 2B). However, some scholars believe that a single intermediate risk factor will not increase the risk of cervical cancer recurrence, but when these intermediate risk factors are combined, the risk of recurrence can increase by $15-20 \%{ }^{20}$. The result of the Laparoscopic Approach to Cervical Cancer Trial has brought further challenges to the establishment of current postoperative adjuvant treatment standards. In terms of 3-year DMFS rates, there were not significant between-group difference among three groups. The results were similar to some previous studies ${ }^{9,21,22}$. Postoperative radiotherapy did not reduce the rate of distant metastasis, which may be related to the biological characteristics of the tumor. Straume $\mathrm{O}$ and Dumoff $\mathrm{KL}$ et al. ${ }^{23,24}$ found that lymphatic vessel density was an important indicator of the prognosis of stage I cervical cancer and a low podo-planin immune-reactivity was associated with lymphatic invasion and lymph node metastasis of cervical cancer. Krishnan J et al. ${ }^{25}$ found that VEGF-C and VEGF-D were involved in mediating the direction of tumor cell migration. In subgroup analysis, patients with stage IB cervical cancer had a higher rate of local control and overall survival after postoperative radiotherapy, revealing that patients with IB stage cervical cancer may be the part of population who benefited from postoperative radiotherapy after laparoscopic hysterectomy. However, no benefits existed in patients with stage IA and IIA1 disease. The possible reasons may be: The sample size was too small to assess the overall survival benefit. The follow-up time of some patients was not long enough to show the differences on OS or DMFS. Furthermore, the 3-year overall survival was already too high to show a between-group difference in subgroup of stage IA disease. It is necessary to further expand the enrolled population and carry out long-term follow-up.

In addition, the main adverse reactions were grade 1-2 acute hematological toxicity and gastrointestinal reactions for patients receiving adjuvant radiotherapy. The study by Porte lance $L{ }^{26}$ showed that the dose distribution for cervical cancer using IMRT was significantly better than those using conventional radiotherapy. In Lin $Y$ et al.'s ${ }^{27}$ meta-analysis, the incidence of genital tract adverse reactions and grade 3-4 adverse reactions in in women receiving intensity-modulated radiotherapy were significantly lower than those receiving conventional radiotherapy or three-dimensional conformal radiotherapy. Therefore, for patients with early stage cervical cancer, postoperative radiotherapy with intensity-modulated technology is safe and effective.

Laparoscopic hysterectomy has been widely used in the past thirty years. Due to its negative impact on survival in the LACC Trial, it is no longer recommended in some cervical cancer guidelines. However, we can't completely deny its clinical benefits. And now laparoscopic hysterectomy is still widely used in other tumors, and whether its negative impact exists in the treatment of other tumors remains to be further investigated. Therefore, laparoscopy should not only be history. We should further explore the mechanism 
of its negative impact on survival, and develop a more suitable adjuvant treatment standards to bring patients better survival and quality of life at the same time.

In conclusion, there is a risk of local failure in laparoscopic hysterectomy for early stage cervical cancer. Adjuvant radiotherapy can reduce the risk of recurrence and improve local control for women with early cervical cancer and bring survival benefits for patients with stage IB disease after minimally invasive hysterectomy.

\section{Declarations}

Acknowledgements[This study was sponsored by the Health and Family Planning Commission of Guangxi Zhuang Autonomous Region (Grant/Award Number: Z20181010).

Author contributions $\square$ Wenqi Liu and Yeping Wei designed this study. Xu-wei Jiang, Xue-mei Xu, Hai-ying Yue, Xiang-de Li, Hui-jun Zhu, Xue Ou, Qiu-lu Zhong collected data and followed the patients. Dan-jing Luo, Qian-fu Liang,Yi-ting Xie performed the data analyses. Li Jiang, Qinghua Du and Yixiu Gan wrote the paper. Wenqi Liu and Jian Li inspected the manuscript critically and took part in the revision of manuscript. Yixiu Gan and Qinghua Du contributed equally to this study and are the co-first authors of this paper. All authors have read and approved the final manuscript.

Conflict of interest: all Authors have nothing to disclose.

\section{References}

1. Ramirez, P. T. et al. Minimally Invasive versus Abdominal Radical Hysterectomy for Cervical Cancer. $N$ Engl J Med. 379 (20), 1895-1904 https://doi.org/10.1056/NEJMoa1806395 (2018).

2. Melamed, A. et al. Survival after Minimally Invasive Radical Hysterectomy for Early-Stage Cervical Cancer. N Engl J Med. 379 (20), 1905-1914 https://doi.org/10.1056/NEJMoa1804923 (2018).

3. Delgado, G. et al. Prospective surgical-pathological study of disease-free interval in patients with stage IB squamous cell carcinoma of the cervix: a Gynecologic Oncology Group study. Gynecol Oncol. 38 (3), 352-357 https://doi.org/10.1016/0090-8258(90)90072-s (1990).

4. Sedlis, A. et al. A randomized trial of pelvic radiation therapy versus no further therapy in selected patients with stage IB carcinoma of the cervix after radical hysterectomy and pelvic lymphadenectomy: A Gynecologic Oncology Group Study. Gynecol Oncol. 73 (2), 177-183 https://doi.org/10.1006/gyno.1999.5387 (1999).

5. Ryu, S. Y. et al. Is adjuvant chemoradiotherapy overtreatment in cervical cancer patients with intermediate risk factors?. Int J Radiat Oncol Biol Phys. 79 (3), 794-799 https://doi.org/10.1016/j.jirobp.2009.11.019 (2011).

6. Koh, W. J. et al. Cervical Cancer, Version 3.2019, NCCN Clinical Practice Guidelines in Oncology. J Natl Compr Canc Netw. 17 (1), 64-84 https://doi.org/10.6004/jnccn.2019.0001 (2019). 
7. Reich, H. New techniques in advanced laparoscopic surgery. Baillieres Clin Obstet Gynaecol. 3 (3), 655-681 https://doi.org/10.1016/s0950-3552(89)80015-x (1989).

8. Nam, J. H. et al. Comparative study of laparoscopico-vaginal radical hysterectomy and abdominal radical hysterectomy in patients with early cervical cancer. Gynecol Oncol. 92 (1), 277-283 https://doi.org/10.1016/j.ygyno.2003.09.003 (2004).

9. Nam, J. H. et al. Laparoscopic versus open radical hysterectomy in early-stage cervical cancer: longterm survival outcomes in a matched cohort study. Ann Oncol. 23 (4), 903-911 https://doi.org/10.1093/annonc/mdr360 (2012).

10. Wang, Y. Z., Deng, L., Xu, H. C., Zhang, Y. \& Liang, Z. Q. Laparoscopy versus laparotomy for the management of early stage cervical cancer. BMC Cancer. 15, 928 Published 2015 Nov 24 https://doi.org/10.1186/s12885-015-1818-4 (2015).

11. Cao, T., Feng, Y., Huang, Q., Wan, T. \& Liu, J. Prognostic and Safety Roles in Laparoscopic Versus Abdominal Radical Hysterectomy in Cervical Cancer: A Meta-analysis. J Laparoendosc Adv Surg Tech A. 25 (12), 990-998 https://doi.org/10.1089/lap.2015.0390 (2015).

12. Sert, B. M. et al. Robot-assisted versus open radical hysterectomy: A multi-institutional experience for early-stage cervical cancer. Eur J Surg Oncol. 42 (4), 513-522 https://doi.org/10.1016/j.ejso.2015.12.014 (2016).

13. Shah, C. A. et al. Surgical and oncologic outcomes after robotic radical hysterectomy as compared to open radical hysterectomy in the treatment of early cervical cancer. J Gynecol Oncol. 28 (6), e82 https://doi.org/10.3802/jgo.2017.28.e82 (2017).

14. Wang, Y. Z., Deng, L., Xu, H. C., Zhang, Y. \& Liang, Z. Q. Laparoscopy versus laparotomy for the management of early stage cervical cancer. BMC Cancer. 15, 928 Published 2015 Nov 24 https://doi.org/10.1186/s12885-015-1818-4 (2015).

15. Frumovitz, M. et al. Comparison of total laparoscopic and abdominal radical hysterectomy for patients with early-stage cervical cancer. Obstet Gynecol. 110 (1), 96-102 https://doi.org/10.1097/01.AOG.0000268798.75353.04 (2007).

16. Kim, S. I. et al. Comparison of survival out- comes between minimally invasive surgery and conventional open surgery for rad-ical hysterectomy as primary treatment in patients with stage IB1IIA2 cervical cancer. Gynecol. Oncol. 153, 3-12 (2019).

17. Kwon, B. S. et al. Dong Soo Suh, Comparison of long-term survival of total abdominal radical hysterectomy and laparoscopy-assisted radical vaginal hysterectomy in patients with early cervical cancer: Korean multicenter, retrospective analysis. Gynecol. Oncol. 159, 642-648 https://doi.org/10.1016/j.ygyno.2020.09.035 (2020).

18. Ebina, Y. et al. Japan Society of Gynecologic Oncology guidelines 2017 for the treatment of uterine cervical cancer. Int J Clin Oncol. 24 (1), 1-19 https://doi.org/10.1007/s10147-018-1351-y (2019).

19. HAIE-MEDER C,MORICE P,CASTIGLIONE et al. Cervical cancer: ESMO Clinical Practice Guidelines for diagnosis,treatment and follow-up [J].Ann Oncol,2010,21(Suppl 5):v37-40. 
20. RYU S Y,KIM M H,NAM B et al. Intermediate-risk grouping of cervical cancer patients treated with radical hysterectomy: a Korean Gynecologic Oncology Group study [J]. Br J Cancer. 110 (2), 278-285 (2014).

21. Lee, E. J., Kang, H. \& Kim, D. H. A comparative study of laparoscopic radical hysterectomy with radical abdominal hysterectomy for early-stage cervical cancer: a long-term follow-up study. Eur $\mathrm{J}$ Obstet Gynecol Reprod Biol. 156 (1), 83-86 https://doi.org/10.1016/j.ejogrb.2010.12.016 (2011).

22. Malzoni, M., Tinelli, R., Cosentino, F., Fusco, A. \& Malzoni, C. Total laparoscopic radical hysterectomy versus abdominal radical hysterectomy with lymphadenectomy in patients with early cervical cancer: our experience. Ann Surg Oncol. 16 (5), 1316-1323 https://doi.org/10.1245/s10434-009-0342-7 (2009).

23. Straume, O., Jackson, D. G. \& Akslen, L. A. Independent prognostic impact of lymphatic vessel density and presence of low-grade lymphangiogenesis in cutaneous melanoma. Clin Cancer Res. 9 (1), 250256 (2003).

24. Dumoff, K. L. et al. Low podoplanin expression in pretreatment biopsy material predicts poor prognosis in advanced-stage squamous cell carcinoma of the uterine cervix treated by primary radiation. Mod Pathol. 19 (5), 708-716 https://doi.org/10.1038/modpathol.3800580 (2006).

25. Krishnan, J. et al. Differential in vivo and in vitro expression of vascular endothelial growth factor (VEGF)-C and VEGF-D in tumors and its relationship to lymphatic metastasis in immunocompetent rats. Cancer Res. 2003 Feb 1;63(3):713 - 22. PMID: 12566318.

26. Portelance, L., Chao, K. S., Grigsby, P. W., Bennet, H. \& Low, D. Intensity-modulated radiation therapy (IMRT) reduces small bowel, rectum, and bladder doses in patients with cervical cancer receiving pelvic and para-aortic irradiation. Int J Radiat Oncol Biol Phys. 51 (1), 261-266 https://doi.org/10.1016/s0360-3016(01)01664-9 (2001).

27. Lin, Y. et al. Intensity-modulated radiation therapy for definitive treatment of cervical cancer: a metaanalysis. Radiat Oncol. 2018;13(1):177. Published 2018 Sep 14. doi:10.1186/s13014-018-1126-7

\section{Figures}




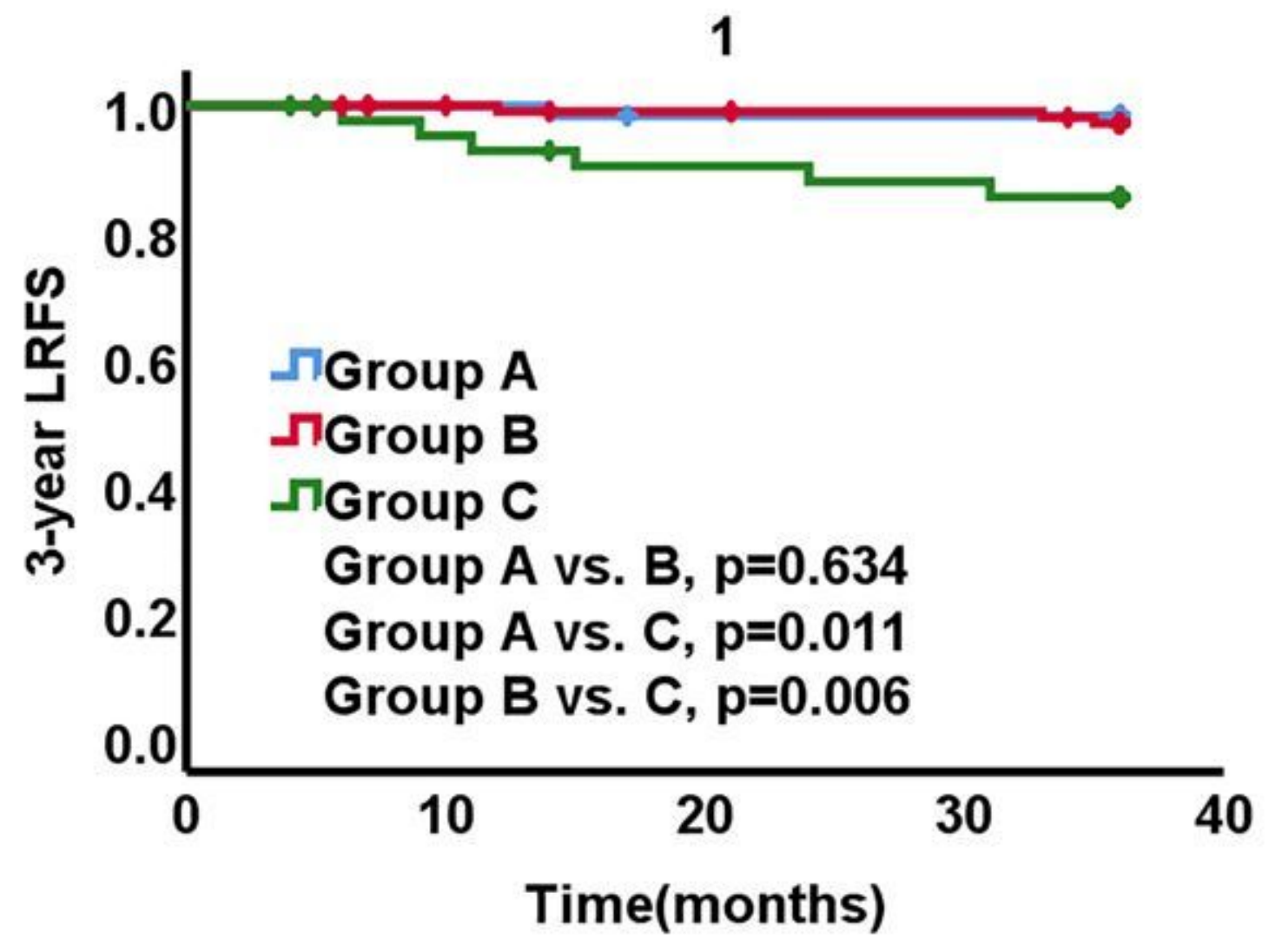

Figure 1

Local recurrence-free survival(LRFS) stratified by three groups. Group A, laparoscopic hysterectomy and adjuvant radiotherapy; Group B, open surgery; Group C, laparoscopic hysterectomy alone. 


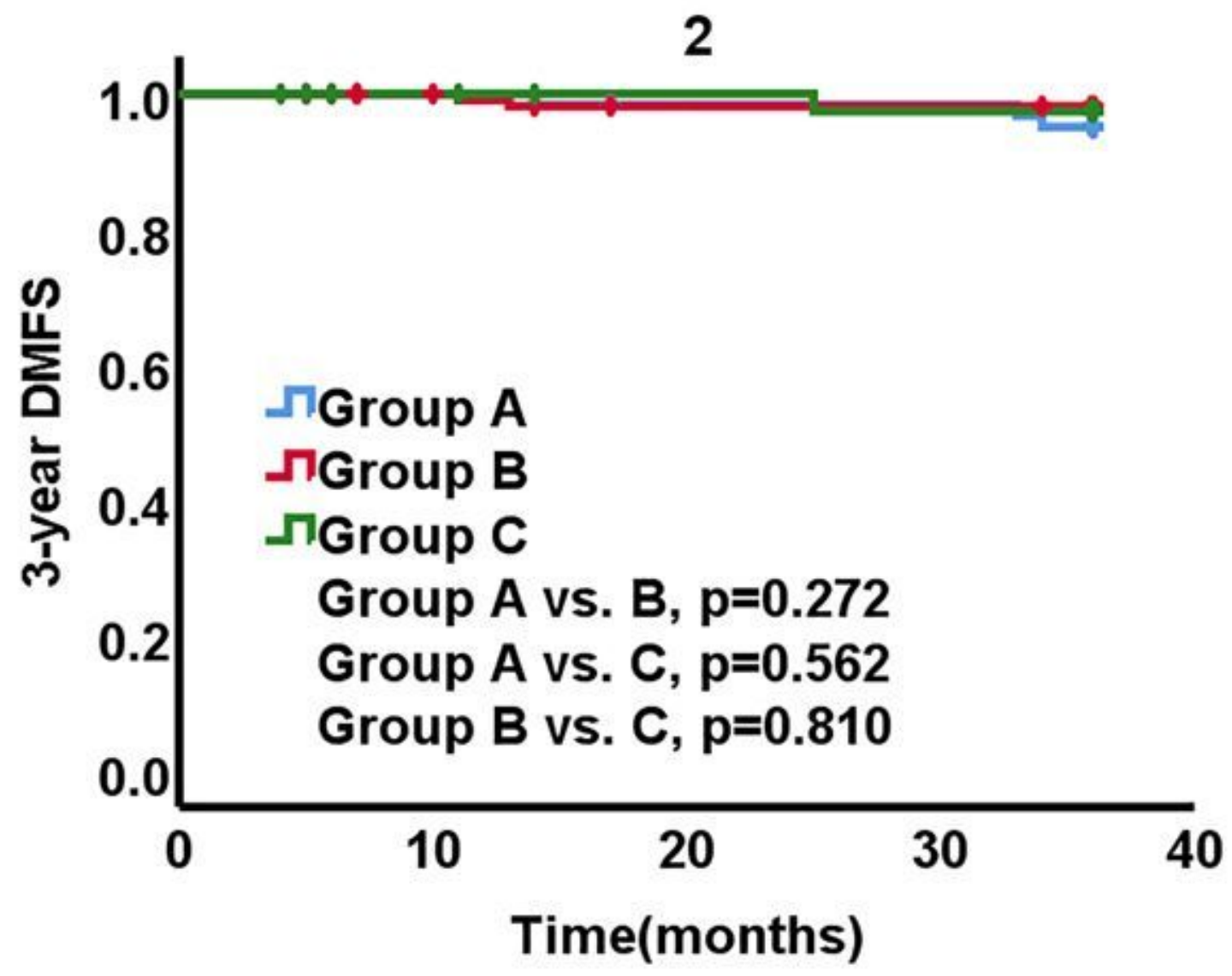

Figure 2

Distant metastasis free survival (DMFS) stratified by three groups. 


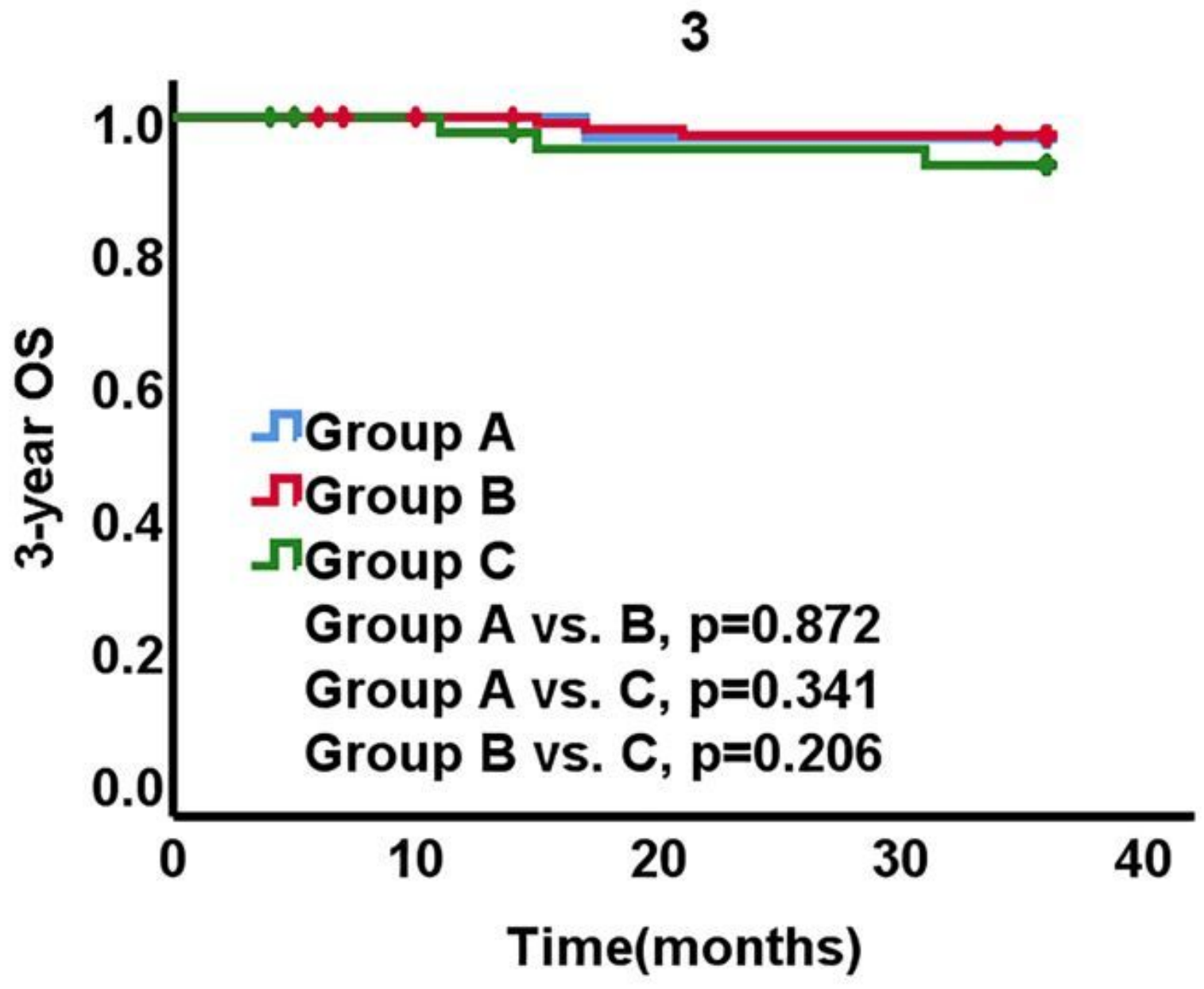

Figure 3

Overall survival (OS) stratified by three groups. 
4

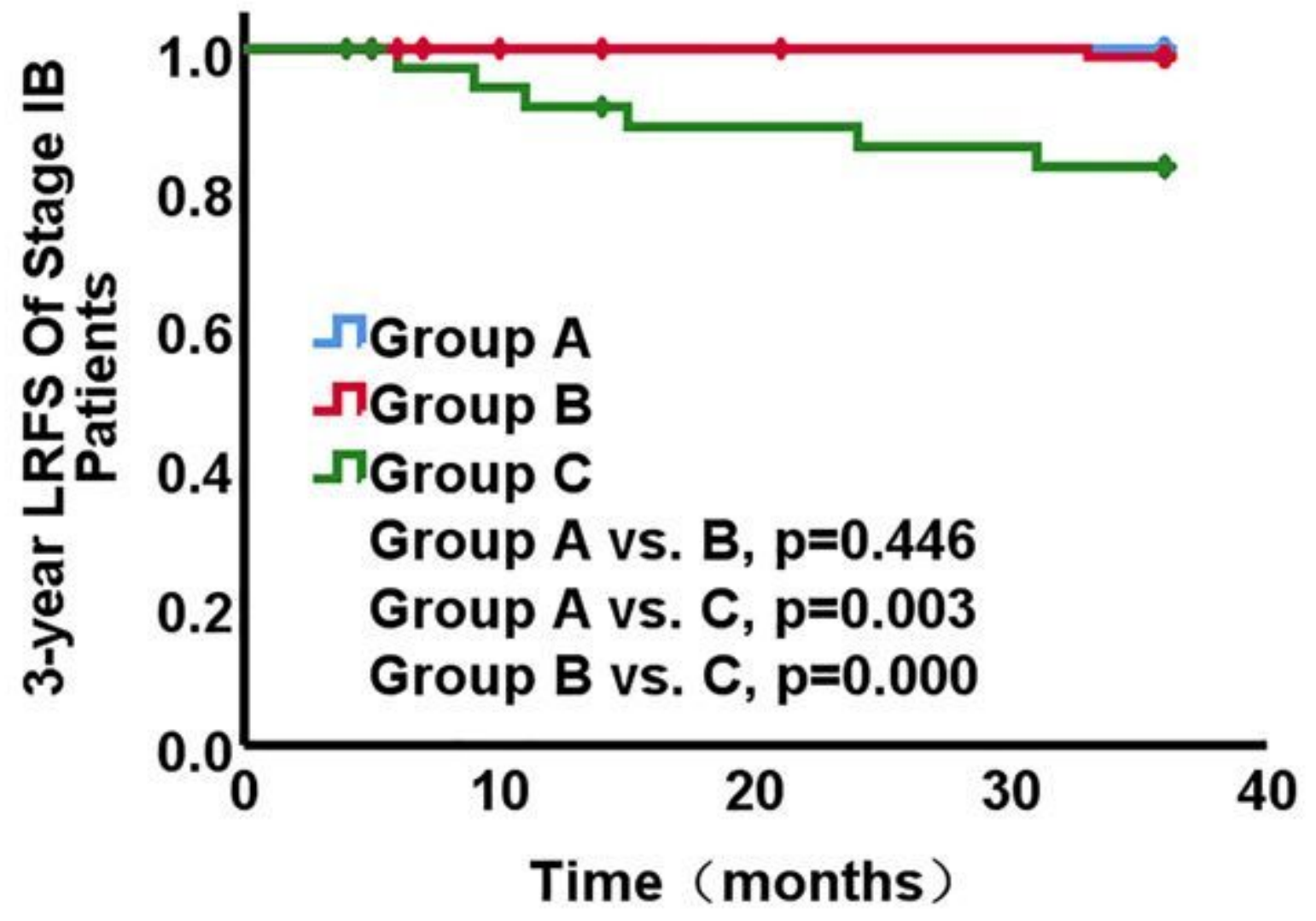

Figure 4

Local recurrence-free survivalखLRFS) stratified by three groups for patients with stage IB1-IB3 disease. 


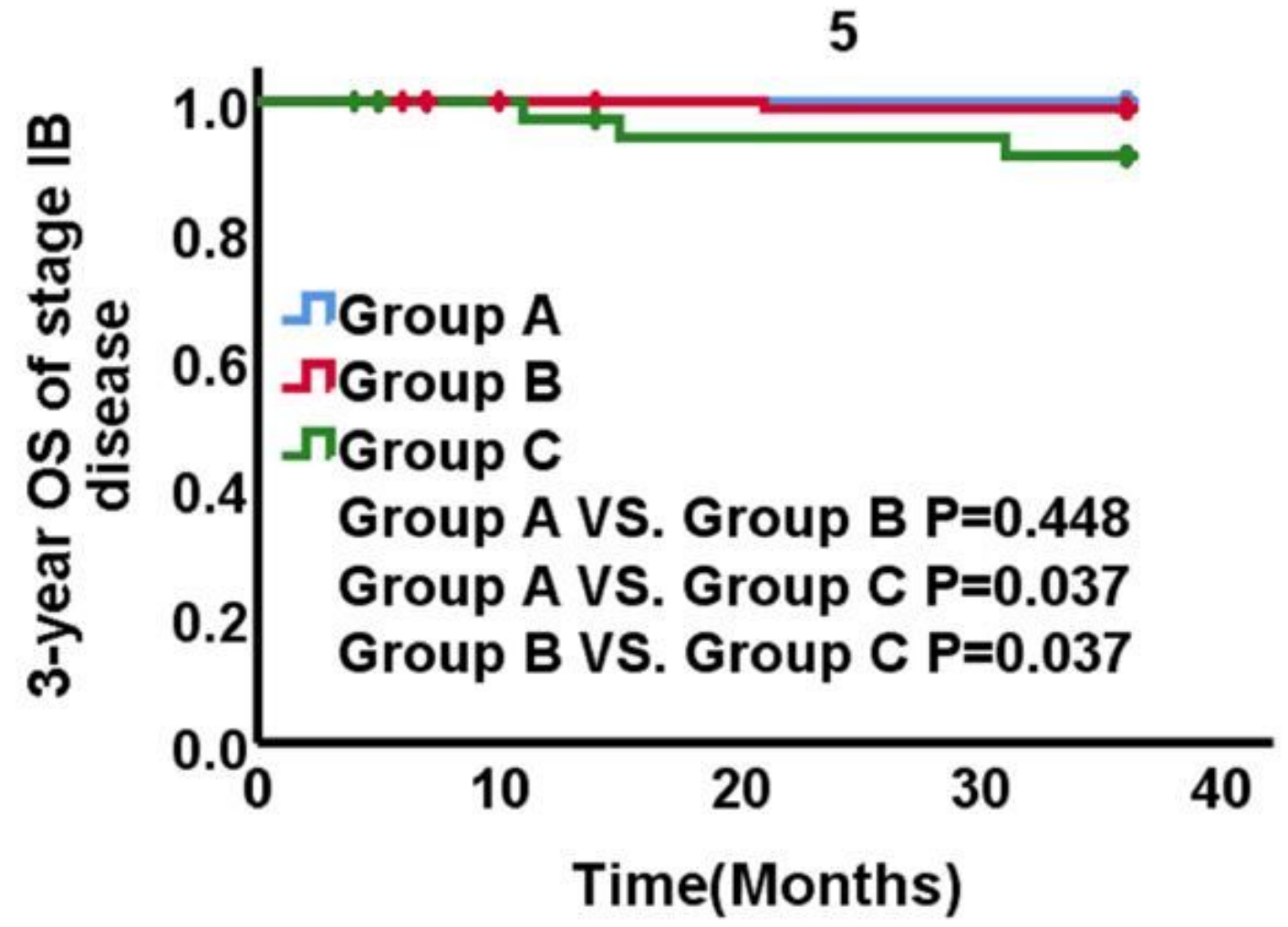

Figure 5

OS stratified by three groups for patients with stage IB1-IB3 disease. 